\title{
Gerenciamento dos riscos assistenciais realizado por enfermeiros em um pronto-socorro
}

\author{
Assistencial risk management performed by nurses in an emergency room
}

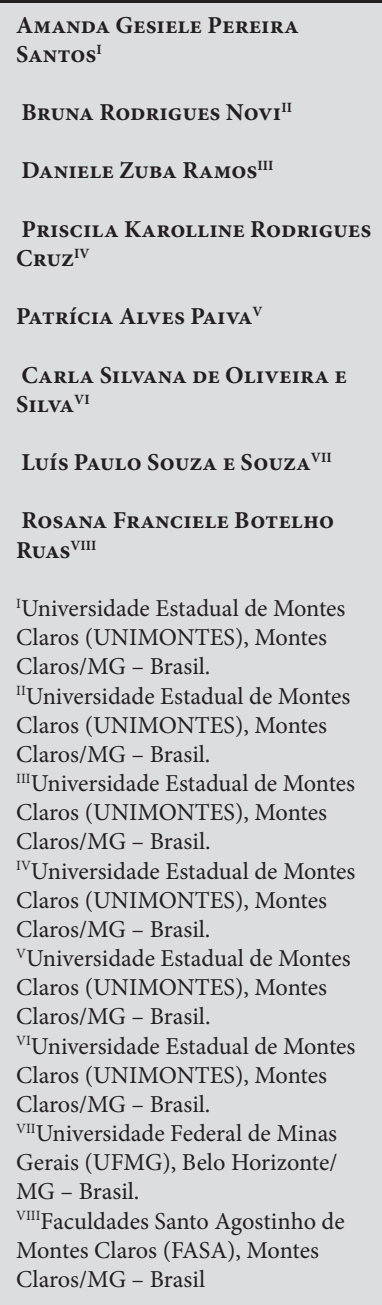

amanda Gesiele Pereira

BRUNA RoDrigues Novi ${ }^{\text {II }}$

DaNiele Zuba Ramos"II

Priscila Karolline Rodrigues
Cruz $^{\text {IV }}$

Patrícia Alves Paiva ${ }^{v}$

Carla Silvana de Oliveira e SILVA $^{\text {VI }}$

Luís Paulo Souza e Souza

Rosana Franciele Botelho

Universidade Estadual de Montes Claros/MG - Brasil.

Claros/MG - Brasil.

"II'Universidade Estadual de Montes

Claros/MG - Brasil.

IvUniversidade Estadual de Montes Claros (UNIMONTES), Montes

Caros/MG - Brasi

Claros (UNIMONTES), Montes

Claros/MG - Brasil.

VIUniversidade Estadual de Montes Claros (UNIMONTES), Montes S/MG - Brasi

Gerais (UFMG), Belo Horizonte/ MG - Brasil.

Montes Claros (FASA), Montes

Claros/MG - Brasil
RESUMo Objetivo: Relatar a experiência da realização do gerenciamento dos riscos assistenciais por enfermeiros de uma Unidade de Pronto-Socorro de um Hospital de ensino.

Métodos: Estudo descritivo, do tipo relato de experiência, realizado em novembro de 2013. A partir de inspeção observacional dos processos assistenciais nas enfermarias, levantaram-se os riscos inerentes aos clientes, classificando-os quanto à frequência. Posteriormente, fez-se a análise dos fatores internos e externos que influenciam no aparecimento dos riscos. O hospital escolhido situa-se na cidade de Montes Claros, norte de Minas Gerais, Brasil.

Resultados: Os resultados encontrados demonstraram um número significativo de riscos assistenciais, emergindo a necessidade da aplicação de instrumentos de gestão. Assim, construiu-se a Matriz SWOT para conhecer os fatores internos e externos que influenciavam o clima organizacional e, consequentemente, o gerenciamento dos riscos.

Conclusão: $\mathrm{O}$ estudo possibilitou identificar que o gerenciamento dos riscos assistenciais realizado pelos enfermeiros na Unidade em questão tem sido um desafio. Entretanto, ficaram evidenciados os benefícios do uso das ferramentas de qualidade e planejamento estratégico na melhoria do cuidado.

Palavras-Chave: Gerenciamento de segurança. Administração HOSPITALAR. EQUIPE DE ENFERMAGEM.

\begin{abstract}
Objective: Reporting the experience of conducting risk management assistance by nurses of emergency department unit of a teaching Hospital. Methods: Descriptive study of type case studies, held in November 2013. From observational assistance processes inspection in the wards, arose the risks to customers, classifying them as the frequency. Subsequently, the analysis of the internal and external factors that influence the appearance of risks. The Hospital is located in the city of chosen Montes Claros, Minas Gerais, Brazil.

Results: The results showed a significant number of social risks, emerging from the need of the application of management instruments. Thus, the SWOT Matrix was built to meet the internal and external factors that influenced the organizational climate and, consequently, the management of risks.

Conclusion: The study made it possible to identify the risk management assistance carried out by nurses in the unit in question has been a challenge. However, were highlights the benefits of using quality tools and strategic planning in improving care.
\end{abstract}

Key-words: Safety Management. Hospital Administration. Nursing TeAm. 


\section{INTRODUÇÃO}

As questões que envolvem a gestão dos recursos administrativos, humanos, materiais e físicos em saúde e em enfermagem estão cada vez mais evidentes no cotidiano de gestores, profissionais e pesquisadores nos estabelecimentos de saúde. A complexidade crescente do processo de produção de cuidados assistenciais exige a reestruturação dos modelos de gestão e das organizações que sejam capazes de fornecer respostas aos desafios encontrados na prestação de serviços pelo Sistema Único de Saúde. Nesse sentido, os enfermeiros têm um papel fundamental em organizar e garantir toda a infraestrutura necessária à assistência a ser prestada por eles e pela equipe multiprofissional. ${ }^{1}$

A crescente demanda de profissionais e aquisição de tecnologias duras, leve-duras e leves nos estabelecimentos de saúde reflete a preocupação em prevenir os riscos à saúde do paciente. Questões como essas ressaltam a necessidade de uma boa gestão do cuidado assistencial e de recursos humanos nas equipes. O gerenciamento de riscos assistenciais surge nesse contexto como importante atividade, uma vez que pretende identificar a provável origem dos eventos, avaliar os danos causados e tomar as decisões apropriadas. ${ }^{1}$ Risco assistencial é aqui definido como os riscos provenientes da prestação dos serviços aos usuários dos serviços de saúde, no que diz respeito à aplicação dos procedimentos prescritos e realizados pela equipe de saúde.

No sistema da gestão organizacional, os tomadores de decisão não precisam mais adivinhar ou copiar outras práticas organizacionais, que muitas vezes são discrepantes da realidade interna da instituição, pois, com as ferramentas do gerenciamento de risco, eles terão fortes evidências de quais práticas serão adequadas à organização, considerando o contexto em que ela esteja inserida. Nesse aspecto, o enfermeiro, como gerente, desenvolve as habilidades de análise de riscos e causas, e a elaboração de estratégias táticas e operacionais para minimização dos riscos, uma vez que possui experiência com o cotidiano das práticas assistências, realiza frequentemente inspeção dos recursos humanos, físicos, materiais e administrativos dos setores de saúde, e tem habilidades e competências intrínsecas à sua formação de gestão clínica e hospitalar. ${ }^{2}$

As competências gerenciais do enfermeiro tornam-se algo desafiador, uma vez que a pluralidade dos aspectos que envolvem instituição, equipe e cliente exige posturas rápidas, assertivas e que sejam organizadas sistematicamente na busca de resultados positivos e efetivos. Ressalta-se nesse contexto o domínio da gestão de pessoas, que tem papel ímpar em uma gestão que apresente bons resultados. ${ }^{2}$

Devido à complexidade dos cuidados assistenciais e do processo de trabalho em saúde, são imprescindíveis modelos eficientes de adequação de pessoal de enfermagem de acordo com o grau de dependência dos pacientes em relação aos cuidados, e estes que sejam condizentes com as condições de trabalho da equipe. O dimensionamento de pessoal tem sido considerado árduo em sua realização, haja vista que esses recursos são os mais complexos da organização, e são responsáveis, ainda, pela mobilização dos demais recursos, como os recursos físicos, materiais e administrativos. ${ }^{1}$ 
Apesar de o enfermeiro possuir um importante papel nos processos de gerenciamento na qualidade do atendimento prestado aos clientes, as dificuldades que esse profissional enfrenta no cotidiano da gestão do serviço são frequentes e pouco exploradas nos estudos na área, de forma que há uma lacuna no sentido de entendimento amplo desses desafios e em propostas de soluções para as dificuldades encontradas nesse contexto. Entre os obstáculos vivenciados no local de trabalho desse profissional constam: falta de segurança à equipe; limpeza e conforto precários; falta de profissionais para o atendimento; elevada demanda de pacientes que poderiam ser atendidos na rede básica de saúde; falta de equipamentos e pouco tempo para executar treinamento à sua equipe. ${ }^{3}$

Há que se destacar que, além de conhecer e minimizar as dificuldades inerentes às atribuições privativas aos enfermeiros, $\mathrm{o}$ mesmo deve, ainda, buscar meios para realizar o gerenciamento da enfermagem de maneira eficiente e eficaz com foco nas necessidades do paciente, conciliando os objetivos da sua equipe com os da organização e, ao mesmo tempo, articular-se no sentido de atender às demandas da equipe multiprofissional. ${ }^{3} \mathrm{O}$ processo de trabalho da equipe de enfermagem exige do enfermeiro a implementação de ações subsidiadas por instrumentos que permitam avaliar os níveis de qualidade dos cuidados prestados.,4 Dessa maneira, justifica-se a necessidade de conhecer as habilidades relacionadas ao gerenciamento de risco do serviço de saúde pelo enfermeiro.

Assim, o objetivo do estudo foi relatar a experiência da realização do gerenciamento dos riscos assistenciais por enfermeiros do pronto-socorro de um hospital de ensino em Minas Gerais, Brasil.

\section{MÉTodos}

Trata-se de estudo descritivo, com abordagem qualitativa, do tipo relato de experiência. O estudo foi realizado em novembro de 2013, no pronto-socorro de um hospital localizado na cidade de Montes Claros, norte de Minas Gerais, Brasil.

Ressalta-se que valores e interesses não entraram em conflito. Quanto aos preceitos éticos, o responsável pelo setor e a administração do referido hospital autorizaram a entrada dos pesquisadores e a realização da observação.

É um hospital que dedica 100\% dos seus leitos para pacientes advindos do Sistema Único de Saúde (171 leitos hospitalares e dez leitos de internação domiciliar).

O pronto-socorro é constituído de 15 leitos, divididos em três salas, que são definidas de acordo com o tipo e tempo de internação dos clientes. O corpo clínico do setor é composto por dois enfermeiros, cinco técnicos de enfermagem, um auxiliar administrativo, um ortopedista, um clínico geral, um cirurgião e um pediatra para cada plantão de 12 horas.

Os dados foram coletados, de forma indireta, levando em consideração as informações de 22 pacientes internados no pronto-socorro. Tais dados foram obtidos por meio das observações feitas nesse cenário, em novembro de 2013, a partir de um roteiro padrão de inspeção utilizado no referido hospital, levando em conta todos os riscos assistenciais, que são todos os riscos que 
podem ocorrer ao paciente decorrentes da prestação da assistência, bem como, em relação à importância e aceitabilidade no que diz respeito ao impacto para a saúde do paciente.

Para este trabalho, foram levantados os riscos de queda, lesão de pele, flebite, trombose, identificação incorreta, risco de infecção e risco para perda de sonda. Foi utilizado para tal um protocolo interno do hospital em estudo. Esse protocolo considera pacientes em risco de queda os com idade acima de 65 anos; glasgow: 14; ramsay: 01; baixa acuidade visual; história pregressa de queda; quimioterapia-heparina intravenosa; déficit de equilíbrio; uso de um grupamento de medicamentos (alguns anti-hipertensivos, opioides, anticonvulsivantes, sedativos, hipnóticos e antiparkinsonianos, que apresentam efeitos colaterais que favorecem a queda). Para a avaliação do risco de lesão de pele, foi utilizada a escala de Braden. ${ }^{5}$ Para flebite, utilizou-se a inspeção visual de sinais flogísticos no local da punção venosa (dor, calor, rubor e edema).

Para risco de trombose, consideraram-se os seguintes critérios: imobilidade no leito (repouso prolongado); anestesia; insuficiência cardíaca, trombose venosa prévia; gravidez; imobilização de membros por gessos e ataduras; politraumatismos (fraturas ósseas); inflamações; cirurgias de grande porte; queimaduras; infarto do miocárdio; insuficiência cardíaca congestiva; idade acima de 40 anos; acidente vascular encefálico; parto e puerpério; e estados de hipercogulabilidade. Para o risco de identificação incorreta, foram observados os critérios de identificação do paciente na cabeceira do leito, na pulseira individual, no prontuário e na abordagem do profissional antes da realização dos procedimentos.
Para o risco de infecção, consideraram-se tempo e aspectos dos dispositivos utilizados no paciente, intra e extracorpóreos, além das condições de vigilância sanitária, tomando como base a Resolução da Diretoria Colegiada 50 da Agência Nacional de Vigilância Sanitária. ${ }^{6}$ Em relação à sonda, avaliou-se a possibilidade de perda, que pode acontecer na movimentação com paciente, transporte ou por falha na fixação, entre outros motivos.

Os riscos pelos quais os clientes estavam submetidos foram classificados em: risco aceitável; moderado; importante; e inaceitável. O risco "aceitável" não necessita de controles adicionais, já o risco considerado como "moderado" exige-se implantação de medidas para reduzir os danos. No risco classificado como "importante", deve-se iniciar uma ação para proceder à redução do risco. Já no risco considerado como "inaceitável", o trabalho não deve ser iniciado, ou deverá ser cortado até serem tomadas medidas de eliminação do risco. ${ }^{7}$

Destaca-se que a análise dos processos administrativos e exame de rotinas do serviço também foi realizada.

A seguir, são apresentados os principais resultados levantados com base nos indicadores usados para classificação. Utilizaram-se números para simbolizar os usuários, prezando pela privacidade dos mesmos.

\section{RESUltados}

No momento das observações, estavam internados na Unidade de Pronto-Socorro 22 pacientes. No Quadro 1, estão representados a relação dos usuários e riscos assistenciais. 
Quadro 1 - Relação de usuários e riscos assistenciais do pronto-socorro de um hospital Universitário de Montes Claros - HUCF. Montes Claros, Minas Gerais, 2013.

\begin{tabular}{|c|c|c|c|c|c|c|c|}
\hline Usuário & $\begin{array}{c}\text { Risco de } \\
\text { Queda }\end{array}$ & $\begin{array}{c}\text { Risco de } \\
\text { Lesão de } \\
\text { Pele }\end{array}$ & $\begin{array}{c}\text { Risco de } \\
\text { Flebite }\end{array}$ & $\begin{array}{c}\text { Risco de } \\
\text { Trombose }\end{array}$ & $\begin{array}{c}\text { Risco de } \\
\text { Identificação } \\
\text { Incorreta }\end{array}$ & $\begin{array}{c}\text { Risco de } \\
\text { Infecção }\end{array}$ & $\begin{array}{c}\text { Risco de } \\
\text { Perda de } \\
\text { Sonda }\end{array}$ \\
\hline $\mathbf{1}$ & 4 & 3 & 3 & 4 & 5 & 4 & 0 \\
\hline $\mathbf{2}$ & 4 & 3 & 3 & 4 & 5 & 4 & 0 \\
\hline $\mathbf{3}$ & 4 & 3 & 3 & 4 & 5 & 4 & 0 \\
\hline $\mathbf{4}$ & 4 & 3 & 3 & 4 & 5 & 4 & 0 \\
\hline $\mathbf{5}$ & 4 & 3 & 3 & 4 & 5 & 4 & 0 \\
\hline $\mathbf{6}$ & 4 & 0 & 3 & 0 & 5 & 4 & 0 \\
\hline $\mathbf{7}$ & 4 & 3 & 3 & 4 & 5 & 4 & 0 \\
\hline $\mathbf{8}$ & 4 & 3 & 3 & 4 & 5 & 4 & 0 \\
\hline $\mathbf{9}$ & 4 & 3 & 3 & 4 & 5 & 4 & 0 \\
\hline $\mathbf{1 0}$ & 0 & 0 & 3 & 0 & 5 & 4 & 0 \\
\hline $\mathbf{1 1}$ & 4 & 0 & 3 & 0 & 5 & 4 & 0 \\
\hline $\mathbf{1 2}$ & 4 & 3 & 3 & 4 & 5 & 4 & 0 \\
\hline $\mathbf{1 3}$ & 4 & 3 & 3 & 4 & 5 & 4 & 0 \\
\hline $\mathbf{1 4}$ & 4 & 3 & 3 & 4 & 5 & 4 & 0 \\
\hline $\mathbf{1 5}$ & 4 & 3 & 3 & 4 & 5 & 4 & 0 \\
\hline $\mathbf{1 6}$ & 4 & 3 & 3 & 4 & 5 & 4 & 0 \\
\hline $\mathbf{1 7}$ & 4 & 3 & 3 & 4 & 5 & 4 & 0 \\
\hline $\mathbf{1 8}$ & 4 & 3 & 3 & 4 & 5 & 4 & 0 \\
\hline $\mathbf{1 9}$ & 4 & 3 & 3 & 4 & 5 & 4 & 0 \\
\hline $\mathbf{2 0}$ & 4 & 0 & 3 & 0 & 5 & 4 & 0 \\
\hline $\mathbf{2 1}$ & 0 & 0 & 3 & 0 & 5 & 4 & 0 \\
\hline $\mathbf{2 2}$ & 0 & 3 & 3 & 4 & 5 & 4 & 0 \\
\hline
\end{tabular}

Legenda: 0: Sem risco; 1: Risco Trivial; 2: Risco Aceitável; 3: Risco Moderado; 4: Risco Importante; 5: Risco Inaceitável.

No Quadro 2, consta a análise da Matriz SWOT do pronto-socorro do estudo a partir do Gerenciamento dos Riscos Assistenciais. A Matriz SWOT é conhecida, também, como Matriz FOFA, por fazer a junção das iniciais das palavras traduzidas para o português (Strengths: Forças; Oportunities: Oportunidades; Weaknesses: Fraquezas; Threats: Ameaças). ${ }^{17}$ Assim, construiu-se a Matriz SWOT da Unidade Hospitalar.

\section{DISCUSSÃo}

Ao analisar o Quadro 1, percebeu-se predominância de riscos assistenciais entre os pacientes. É importante destacar que o setor recebe pacientes de diferentes complexidades, aspecto influenciador no aumento da possibilidade de risco devido à característica intrínseca da fisiopatologia das doenças e estado de comprometimento da saúde. Entretanto, não se deve deixar de descrever, todavia, riscos inerentes à assistência e gestão dos serviços, pelo alto volume de atendimentos e agilidade necessária.

No que diz respeito ao "risco de identificação incorreta", este foi evidenciado em todos os pacientes. A identificação correta é um procedimento que tem como objetivo assegurar a prevenção de erros e danos 
que possam lesar o paciente. Nesse sentido, várias medidas podem ser adotadas como: identificação na cabeceira do leito; conferência verbal com o paciente; sendo a forma mais segura a pulseira de identificação. ${ }^{8}$ Reforça-se que a associação das medidas tende a garantir maior segurança na prestação dos cuidados. ${ }^{8}$

O risco de identificação incorreta é considerado inaceitável, conforme protocolo interno do hospital, uma vez que pode facilitar a troca de medicações e de procedimentos invasivos, podendo acarretar lesão permanente ou óbito ao paciente. Ressalta-se que esse risco é modificável, já que parte da capacidade de gestão e mobilização da equipe em estabelecer uma série de critérios de conferência que o minimize. ${ }^{9}$

Outro risco presente em $100 \%$ dos pacientes internados foi o de "infecção". Serão consideradas preveníveis aquelas infecções que podem ser evitadas por meio da interferência na cadeia de transmissão do microrganismo, a partir da utilização de medidas de precaução, por exemplo. Já as classificadas como não evitáveis são aquelas que, ainda que sejam utilizadas as precauções padrão, a infecção se instala. Tais infecções são observadas em paciente com comprometimento do sistema imune, originárias de sua microbiota. Autores afirmam que aproximadamente $30 \%$ das infecções são consideradas evitáveis, o que exige da equipe de saúde e das instituições responsabilidade ética, técnica e social na prevenção dessas infecções no processo de cuidar. ${ }^{8}$

$\mathrm{O}$ "risco de queda" foi observado em $81,8 \%$ dos pacientes, sendo considerado importante pela possibilidade em gerar lesão ou perda de função permanente. As quedas

Quadro 2 - Análise SWOT do Pronto-Socorro do Hospital Universitário de Montes Claros, a partir do Gerenciamento dos Riscos Assistenciais. Minas Gerais, 2013.

\begin{tabular}{|c|c|c|}
\hline \multicolumn{3}{|c|}{ ANÁLISE SWOT } \\
\hline \multirow[b]{2}{*}{$\begin{array}{l}\text { FATORES } \\
\text { INTERNOS }\end{array}$} & Pontos Fortes & Pontos Fracos \\
\hline & $\begin{array}{l}\text { - Hospital que preza pelo ensino e } \\
\text { pesquisa. } \\
\text { - Hospital referência em doenças } \\
\text { infecto- contagiosas. } \\
\text { - Hospital Amigo da Criança. } \\
\text { - Dispõe do Programa Hospital em Casa. } \\
\text { - Apresenta o Programa Doutores da } \\
\text { Alegria. }\end{array}$ & $\begin{array}{l}\text { - Estrutura física inadequada. } \\
\text { - Quantidade insuficiente de leitos. } \\
\text { - Quantidade insuficiente de colaboradores } \\
\text { para a assistência. } \\
\text { - Gestão Ineficaz. }\end{array}$ \\
\hline \multirow[b]{2}{*}{$\begin{array}{c}\text { FATORES } \\
\text { EXTERNOS }\end{array}$} & Oportunidades & Ameaças \\
\hline & $\begin{array}{l}\text { - Títulos de Acreditação Hospitalar nos } \\
\text { três níveis; } \\
\text { - Grupos de apoio ao hospital; } \\
\text { - Recursos Governamentais: "Pró-Hosp... }\end{array}$ & $\begin{array}{l}\text { - Concorrência; } \\
\text { - Perda de recursos governamentais; } \\
\text { - Perda do processo de acreditação hospitalar. }\end{array}$ \\
\hline
\end{tabular}


são os eventos adversos mais frequentes no contexto hospitalar, sendo responsáveis por aumento no período de internação e acréscimo nos custos econômicos e sociais. Autores afirmam ${ }^{10}$ que a queda acontece em $2 \%$ a $15 \%$ dos pacientes hospitalizados, e mais de um quarto dessas resultam em lesões físicas.

Em relação ao "risco de lesão de pele", este foi observado em $77,2 \%$ dos pacientes. Foi considerado moderado pela possibilidade de perda de função provisória do tecido e pela necessidade de implementação de medidas no sentido de prevenção. Entre as lesões de pele, as que são consideradas de maior impacto são as úlceras por pressão (UP). Estudos apontam ${ }^{11}$ que 95\% das UP são evitáveis, o que reforça a necessidade de gerenciamento desse risco.

Observado em 100\% dos pacientes, o "risco para flebite" foi considerado moderado, uma vez que necessita de controles adicionais para evitar a instalação de um processo infeccioso, especialmente no local da punção venosa. A flebite é uma das complicações infecciosas mais comuns relacionadas ao uso de cateter venoso periférico. $\mathrm{O}$ uso do cateter é prática diária para a administração de soluções e requer cuidados na inserção, manutenção, salinização, troca e descarte. A adoção das precauções padrão garante a segurança do paciente e do profissional, e pode minimizar o aparecimento de flebites nos sítios de inserção dos cateteres. A prevenção e a detecção precoce das complicações provenientes da terapia endovenosa são atribuições do enfermeiro. ${ }^{12}$

O "risco de trombose", por sua vez, foi encontrado em $77,2 \%$ dos pacientes, tendo sido considerado como importante, uma vez que, ao ser identificado, exige-se a im- plantação de medidas de precaução imediatas. A trombose venosa profunda é frequentemente uma complicação cirúrgica e clínica, mas pode ocorrer em pacientes hígidos. A estimativa da incidência da trombose venosa no Brasil é de 0,6 casos por 1.000 habitantes/ano, sendo um estado que pode levar à complicações graves como a embolia pulmonar e síndrome pós-trombótica. ${ }^{13}$

$\mathrm{O}$ "risco para perda de sonda" foi observado em apenas um dos 12 pacientes que usavam sonda, e foi considerado como risco moderado.

Ao analisar os riscos assistenciais pelos quais os pacientes estavam expostos, é importante reforçar que as condições de saúde apresentadas por esses pacientes predispuseram o aumento dos riscos encontrados. Entretanto, a literatura é consistente quanto ao fato de que a execução da assistência também tem papel determinante na manifestação desses riscos..$^{9,10,11,12}$

Um fator ímpar para a efetivação dos riscos assistenciais é a gestão de pessoas. Em um ambiente complexo onde os riscos são multifatoriais, a equipe tem que estar alinhada e focada nas medidas preventivas. Entretanto, fatores como clima organizacional e os modos de organização do trabalho (baixa quantidade de mão de obra distribuída entre o número de pacientes, por exemplo) favorecem a sobrecarga no trabalho, podendo levar a maior ocorrência de erros e riscos ao paciente. ${ }^{14}$

No contexto do estudo, identificaram-se aspectos que poderiam favorecer o aumento dos riscos relacionados à assistência, como: condições de trabalho (carga horária); quantidade de profissionais para a alta demanda; fatores que influenciam no desem- 
penho/desgaste profissional. Considerando esses aspectos, realizou-se o dimensionamento do pessoal de enfermagem, segundo a Resolução 293/04 do Conselho Federal de Enfermagem e utilizando a carga horária de 30 horas semanais como referência. ${ }^{15}$

Aplicou-se o escore para a classificação quanto ao grau de dependência dos pacientes. Em seguida, utilizou-se a fórmula de cálculo do Fugulin para quantificação. ${ }^{1}$ Como resultado, obteve-se o total de 34 funcionários, sendo: dez enfermeiros e 24 técnicos em enfermagem.

De acordo com o cálculo, foi possível perceber que o setor não é assistido de maneira adequada pela equipe de enfermagem, fator que pode influenciar no aumento da probabilidade da ocorrência de riscos. Autores $^{1}$ esclarecem que mesmo reconhecendo a importância dos recursos humanos na qualidade da assistência, as lideranças de enfermagem encontram grandes resistências para adequar o número de pessoal à demanda de atendimento nas instituições de saúde, principalmente em razão de justificativas orçamentárias.

O processo de trabalho em um pronto-socorro é intenso, assim, o dimensionamento do pessoal de enfermagem é fundamental para uma assistência que vise à segurança do paciente e dos colaboradores, assim como à excelência na qualidade. ${ }^{16}$

Ao avaliar a demanda do serviço em estudo e quantidade de profissionais de enfermagem, surgiu a necessidade de averiguar outros potenciais fatores que influenciavam a gestão de pessoas e, consequentemente, $o$ gerenciamento de risco. Sendo assim, emergiu a necessidade de avaliação mais profunda dos aspectos internos e externos que in- fluenciavam no clima organizacional. Como importante ferramenta para esse tipo de análise, tem-se a Matriz SWOT, a qual recebe esse nome por fazer a junção das iniciais das palavras em inglês "Strengths", "Weaknesses", "Opportunities" e "Threats". ${ }^{17}$ Essa ferramenta demonstra ser útil para verificar a posição estratégica da empresa. Refere-se a uma avaliação global dos pontos fortes e dos pontos fracos da organização à luz das oportunidades e das ameaças em seu ambiente. ${ }^{17}$ Assim, a partir da análise global da organização, foi possível identificar fraquezas e ameaças que podem representar pressões e condicionantes para a dificuldade do gerenciamento eficaz do risco assistencial no pronto-socorro, podendo citar: falta de estrutura física adequada; insuficiência de leitos; número insuficiente de colaboradores.

Percebeu-se a necessidade de uma elaboração de estratégias que potencializem os pontos fortes, além de minimizar os pontos fracos para o alcance das oportunidades e $o$ afastamento das ameaças.

A Matriz retornou para o serviço, com vistas a contribuir para que os gestores da Unidade e do Hospital pudessem dar prosseguimento às medidas de melhoria.

\section{CONSIDERAÇões Finais}

Conclui-se que os riscos mais predominantes foram os de infecção, de identificação e de flebite, observados em todos os pacientes do pronto-socorro. Tal cenário possibilitou avaliar que a experiência de gerenciamento de riscos assistenciais realizados por enfermeiros na Unidade de pronto-socorro em estudo é um desafio, pois existem fatores internos e externos influenciadores na deficiên- 
cia do gerenciamento de risco, na gestão do cuidado e das pessoas do setor. Esses fatores puderam ser explicitados pelo planejamento estratégico utilizado. Portanto, este estudo sugere a força de tal ferramenta na otimização dos pontos fortes e minimização dos pontos fracos, destacando que os resultados podem e devem ser usados para reorganizar o modelo assistencial dos profissionais de enfermagem e, consequentemente, redução dos riscos relacionados à assistência.

\section{REFERÊNCIAS}

1. Magalhães ANM, Riboldi CO, Dall'agnol CM. Planejamento de recursos humanos de enfermagem: desafio para as lideranças. Rev Bras Enferm. 2009; 62(4): 608-12.

2. Ruthes RM, Cunha ICK. O. Competências do enfermeiro na gestão do conhecimento e capital intelectual. Rev Bras Enferm. 2009; 62(6): 901-5.

3. Bellucci Júnior JÁ, Matsuda LM. O enfermeiro no gerenciamento à qualidade em serviço hospitalar de emergência: revisão integrativa da literatura. Rev Gaúcha Enferm. [internet]. 2011 [citado 2014 set. 10]; 32(4):797-806. Disponível em: http://www.scielo.br/pdf/rgenf/v32n4/ v32n4a22.pdf

4. Kurcgant P., Tronchin DMR, Melleiro MM. A construção de indicadores de qualidade para a avaliação de recursos humanos nos serviços de enfermagem: pressupostos teóricos. Acta Paul Enferm. 2006; 19(1):88-91.

5. Serpa LF, Santos VLCG, Campanili TCGF, Queiroz M. Validade preditiva da escala de Braden para o risco de desenvolvimento de úlcera por pressão, em pacientes críticos. Rev Latino-Am Enfermagem. 2011;19(1): 50-7.

6. Agência Nacional de Vigilância Sanitária. Resolução - RDC no . 50, de 21 de fevereiro de 2002. Dispõe sobre o regulamento técnico para planejamento, programação, elaboração e avaliação de projetos físicos de estabelecimentos assistenciais de saúde. Brasília, 2002.

7. Feldman LB. Como alcançar a qualidade nas instituições de saúde: critérios de avaliações, procedimentos de controle, gerenciamento de riscos hospitalares até a certificação. São Paulo: Martinari; 2004.

8. Carvalho VT, Cassiani SHB. Erros na medicação: Análise das situações Relatadas pelos profissionais de enfermagem. Medicina. 2000; 33:322-30.

9. Pereira MSP, Souza ACS, Tripple AFV, Prado MA. A infecção hospitalar e suas implicações para o cuidar da enfermagem. Texto Contexto Enferm. 2005; 14 (2): 250-7.

10. Almeida RAR, Abreu CCF, Mendes AMOC. Quedas em doentes hospitalizados: contributos para uma prática baseada na prevenção. Rev Enferm Referência. 2010; 2(2): 163-72.

11. Louro M., Ferreira M., Povoa P. Avaliação de Protocolo de Prevenção e Tratamento de Úlceras de Pressão. Rev bras ter intensiva. 2007; 19(3): 337-41.

12. Moncaio ACS, Figueiredo RM. Conhecimentos e práticas no uso do cateter periférico intermitente pela equipe de enfermagem. Rev Eletr Enf [internet]. 2009 [citado 2014 set. 10]; 11(3): 620-7. Disponível em: http://www.fen.ufg.br/revista/v11/n3/v11n3a20.htm

13. Garcia ACF, Souza BV, Volpato DE, Deboni LM, Souza M V, Martinelli R, et al. Realidade do uso da profilaxia para trombose venosa profunda: da teoria à prática. J Vasc Br. 2005;4(1): 35-41.

14. Balsanelli AP, Jericó MC. Os reflexos da gestão pela qualidade total em instituições hospitalares brasileiras. Acta Paul Enferm. 2005; 18(4): 397-402. 
15. Conselho Federal de Enfermagem. Resolução 293/2004: fixa e estabelece parâmetros para o dimensionamento do quadro de profissionais de enfermagem nas unidades assistenciais das instituições de saúde e assemelhados. Rio de Janeiro, 2004.

16. Ohara R., Melo MRAC, Laus AM. Caracterização do perfil assistencial dos pacientes adultos de um pronto socorro. Rev Bras Enferm. 2010; 63(5): 749-54.

17. Souza e Souza LP, Souza AMV, Pereira KG, Figueiredo T., Bretas TCS, Mendes, MAF, et al. Matriz Swot como ferramenta de gestão para melhoria da assistência de enfermagem: estudo de caso em um hospital de ensino. Gestão \& Saúde [internet]. 2013 [citado 2014 set. 10]; 4(1): 1911-21. Disponível em: http://gestaoesaude.unb.br/index.php/gestaoesaude/article/view/415/pdf

\section{DAdos dos Autores}

\section{Amanda Gesiele Pereira Santos}

Graduada em Enfermagem pela Universidade Estadual de Montes Claros. Trabalha na instituição Corpo de Bombeiros Militar de Minas Gerais. Montes Claros/MG - Brasil. nandagesiele@yahoo. com.br

\section{BRUna Rodrigues Novi}

Graduada em Enfermagem pela Universidade Estadual de Montes Claros. Montes Claros/MG - Brasil.bruna_novi@hotmail.com

\section{Daniele Zuba Ramos}

Graduada em Enfermagem pela Universidade Estadual de Montes Claros. Montes Claros/MG - Brasil.danielezuba@yahoo.com.br

\section{Priscila Karolline Rodrigues Cruz}

Graduada em Enfermagem pela Universidade Estadual de Montes Claros. Montes Claros/MG - Brasil.karolline_rcruz@yahoo.com.br

\section{Patrícia Alves Paiva}

Mestranda em Enfermagem pela Universidade Estadual de Montes Claros. Montes Claros/MG - Brasil. patricia.alves.paiva@hotmail.com

\section{Carla Silvana de Oliveira e Silva}

Doutora em Enfermagem pela Universidade Estadual de Montes Claros. Professora do Departamento de Enfermagem. Montes Claros/MG - Brasil. carlasosilva@ig.com.br

\section{Luís Paulo Souza e Souza}

Doutorando em Saúde Pública pela Universidade Federal de Minas Gerais Professor do Departamento de Medicina. São João del Rei/MG - Brasil. luis.pauloss@hotmail.com

\section{Rosana Franciele Botelho Ruas}

Mestra em Enfermagem pela Faculdades Instituto Prominas Montes Claros. Professora do Departamento de Enfermagem. Montes Claros/MG - Brasil. rosanaruas@yahoo.com.br

Submetido em:7-9-2016

Aceito em: 5-4-2017 